\title{
XXVII.
}

Aus der chirurgischen Abtheilung des Herzoglichen Krankenhauses zu Braunschweig (Prof. Sprengel).

\section{Ein Fall von Soorinfection als Beitrag zur Pathogenese des Soor. \\ von}

Dr. Denecke,

Oberarzt des Krankenhauses St. Marienberg zu Helmstedt, früher Assistent der Abtheilung.

(Mit 8 Abbildungen.)

In meiner Arbeit: „Ueber die Entzündung des Meckel'schen Divertikels und die Gangrän desselben " hatte ich bereits kurz erwähnt, dass in dem von uns beobachteten Fall von Entzündung des Divertikels, in dem es zur Perforation gekommen war, ein Fadenpilz als Ursache derselben gefunden wurde. Es hatte sich um einen Knaben gehandelt, der 4 Tage vor der Aufnahme plötzlich mit Sehmerzen im Leibe und Erbrechen erkrankt war. Er wurde unter ausgesprochenen Erscheinungen einer circumscripten Peritonitis aufgenommen mit deutlich fühlbarer Resistenz nach innen von der Ileocöcalgegend.

Bei der Operation fand sich als Ursache der Peritonitis eine Perforation des Divertikels, um das sich allseitig Darmsehlingen herumgelegt hatten; besonders bildete die Flexur mit ibren stark verdickten Appendices ein grosses Convolut, innerhalb dessen sich etwas zäher Eiter fand. Es wurde die Resection des Divertikels mit Uebernähen der Darmwunde vorgenommen; doch starb das Kind am Tage nach der Operation.

Beobachtungen über pathogene Wirkung von Fadenpilzen beim Menschen sind bisher nur ganz vereinzelt gemacht worden, und so dürfte der vorliegende Fall um so mehr zur genauen Beschreibung des morphologischen Verhaltens des Pilzes, sowie der durch ihn hervorgerufenen pathologisch-anatomischen Veränderungen geeignet sein, als der Tod des betreffenden Menschen durch diesen Fadenpilz herbeigeführt wurde. 
Ich lasse zunächst das Sectionsprotok oll (Prof. Dr. Beneke) folgen:

In der rechten Regio iliaca reizlose Laparotomiewunde: im oberen und unteren Wundwinkel Jodoformgazetampons. Der untere reicht zwischen Blase und Beckenrand bis fast zum Rectum abwärts. Das umgebende Bindegewebe ist von einer mässigen Menge serös-eitriger Flüssigkeit diffus infiltrirt. Der Tampon der oberen Wundecke reicht etwas median an eine Darmwunde des Ileum, die etwa in der Mitte zwischen Nabel und Blasenapex liegt. Der Tampon ist fast trocken, nicht blutig; die ihm anliegenden Theile der Serosa geröthet, aber vollkommen trocken. Die weitere Umgebung zeigt weiche, frische Verklebung der Darmschlingen durch ödematös-fibrinöses Exsudat, welches stellenweise einen fast rein eitrigen Charakter hat. Ueber die Medianlinie reicht die Verklebung nicht hinaus, desgleichen ist die Leberumgebung frei von Exsudat. Im Douglasraum kein freier Eiter; hier sind die Schlingen des S. romanum mit ihren tiefroth injicirten, verdickten Appendices zu einem dicken, harten Ballen verklebt, doch lassen sich alle Theile leicht auseinander ziehen. Die Darmschlingen sind thberall leicht meteoristisch gebläht, blass, nicht schwappend. - Im Magen und Iejunum nichts Besonderes; im Ileum kurz vor der Operationsstelle, welche etwa dem Orte eines Divertikels entsprechen würde, einige kleine frischrothe Coagula, desgleichen unterhalb der Operationsstelle. Colon ohne Befund. Die Subserosa des S. romanum und der Appendices sind von rahmigem Eiter stark infiltrirt, derselbe fliesst von der Schnittfläche in dicken Tropfen ab. Die Darmwandung erreicht an diesen Stellen theilweise die Dicke von $2-3 \mathrm{~cm}$; zwischen kleineren, bis hanfkorngrossen Abscessen liegt ein hämorrhagisch infiltrirtes, lockeres, von einigen Fetträubchen durchsetztes Gewebe. Die Muscularis ist hypertrophisch, die Schleimhaut mässig ödematös, blass, mit reichlichem, dickglasigem Schleim bedeckt. Rectum ohne Befund. Blasenschleimhaut etwas ödematös, zeigt einige Ecchymosen. Wenige Tropfen traben Harns im Lumen. - Milz gross, dunkelroth, fest, trocken, nicht quellend, nicht trüb. - Nebennieren und Nieren ohne Befund, letztere etwas blass. - In der Leber keine Herde. Am Cöcum liegen zwei völlig verkäste Lymphdrüsen, die eine kirschkern-, die andere kirschgross; das käsige Material weich breiig. - Linke Lunge nicht verwachsen, lufthaltig, streckenweise geringes interstitielles Emphysem. Im Unterlappen ein grösserer Abschnitt bronchopnenmonischer Infiltration mit schwerer eitriger Bronchitis. Trachea, Larynx, Oesophagus und Magen ohne Besonderheit.

In einem Deckelglastrockenpräparat, zu dem von den Darmbelägen etwas Secret entnommen war, fanden sich ausser Eiterkörperchen eine grosse Anzahl von den Hefezellen durchaus gleichenden Gebilden der verschiedensten Grösse, die in Haufen zusammenliegen und auch kleine Sprossverbände zeigen. Irgendwelche andere Mikroorganismen werden vermisst. Sofort nach der Section wurde ferner von Herrn Professor Dr. Beneke etwas Material auf Agar ubertragen, und es gingen hier sehr üppig Reinculturen jener vermeintlichen Hefe auf.

Das Divertikel selbst, dessen Beschreibung ich hier einschalte, war 
etwa 5-6 cm lang. Es hat im Ganzen die Form einer Birne, deren engerer Theil dem Darm aufsass. Zwischen diesem und dem ilm aufsitzenden, fast kegelförmigen Körper bemerkt man die unregelmässige, trichterförmige Perforationsöffnung, die mit schmutzig gelblichem Belag

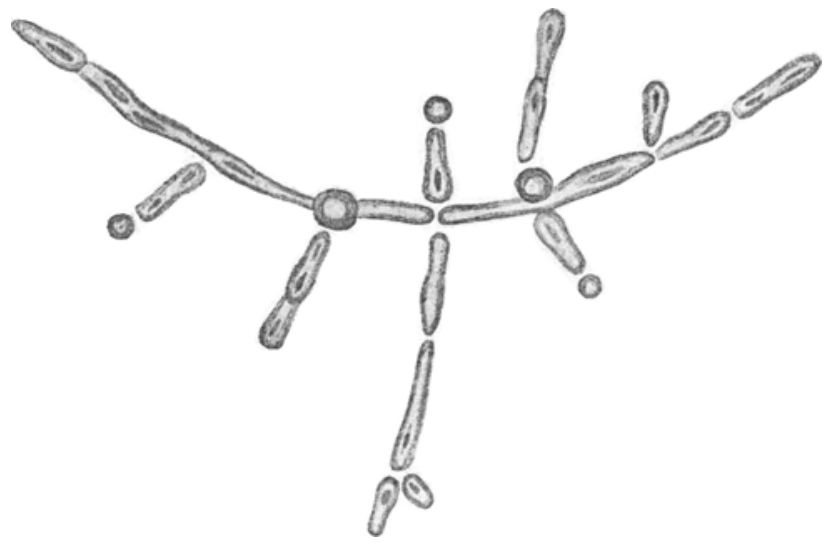

Fig. 1.

bedeckt ist. Im Uebrigen ist die Oberfläche vollkommen glatt, abgesehen von geringen fibrinösen Auflagerungen. Von der Resectionswunde aus gelangt man mit der Sonde leicht in die Perforationsöffnung, dagegen ge-

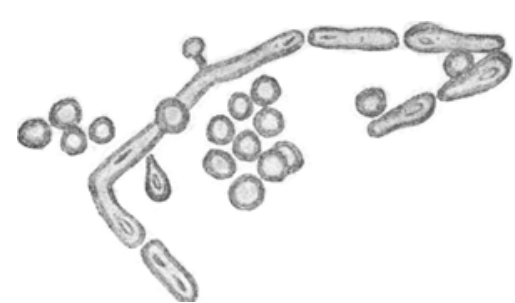

Fig. 2.

lingt es erst nach einigem Suchen, einen feinsten Gang zu finden, der das Lumen des Halses mit dem des Körper verbindet. Auf einem durch diesen Gang und die Perforationsstelle gelegten Längsschnitt sieht man zunächst die Innenseite des Halses von normaler Schleimhaut bedeckt. $\mathrm{Z}$ wischen ihm und dem Körpers, der mit einem klaren, dicken Schleim gefült ist, liegt das perforirte, tiefe Geschwilr, das mehr als die Hälfte des Querschnittes einnimmt. Es hat einen schmierigen, gelben Belag, in dem man mikroskopiseh eine grosse Anzahl verschieden langer dicker Pilzfăden sieht, die sich durch ihr starkes Lichtbrechungsvermögen leicht erkennen lassen. Die Fäden bestehen aus einer Anzahl scharf von einander getrennter Einzelglieder, die zumal an dem Ende des Fadens oft kolbig aufgetrieben sind und hier bisweilen eine Conidie tragen. Von dem Hauptfaden gehen nach allen Richtungen kleinere Seiten$\mathrm{zweige} a b$, die in ihren Anfängen aus einer Seitenknospe bestehen (Fig. 1). Die Conidienform des Pilzes (Fig. 2) - denn dass beide Formen nur verschiedene Entwicklungsstufen desselben Pilzes seien, schien von vorn herein klar - tritt in dem Geschwür gegen die Fadenform zurtlck, doch kommen einzelne und auch zu kleineren Haufen zusammenliegende Coni- 
dien vor, die durch einen sie umgebenden, stark lichtbrechenden Ring ausgezeichnet sind.

Die nächste Umgebung des Geschwurs wird von einem derben, schwieligen, bis zu ${ }^{3} / 4 \mathrm{~cm}$ dicken Gewebe gebildet, das peripher ganz allmählich an Dicke abnimmt, während es den feinen, Hals und Körper verbindenden Gang allseitig umschliesst. Zwischen dem geschwärigen Belag und diesem Schwielengew ebe liegt eine mehrere Millimeter dicke Zone von jungem, blutreichen Gewebe. Die Wandung des ampullenförmig ausgedehnten Körpers des Divertikels ist sehr ungleich dick, sie nimmt vom Halstheil an allmählich $a b$, ist an dem freien Ende des Körpers fast papierdün; sie ist innen von einer zarten, abhebbaren Epithelschicht bedeckt, in der vereinzelt Conidien liegen. Der schleimige Inhalt des Körpers enthält reichlich Pigment.

Hatten wir schon in Zupfpräparaten von dem Belag des Geschwtirs lange, sprossartig verzweigte Fäden gesehen, so finden sich dieselben noch reichlicher in Schnittpräparaten, an denen man gleichzeitig ihr Verhalten zu dem Gewebe studiren kann. Die Schnitte wurden, soweit die Pilze sichtbar werden sollten, nach Gram gefärbt mit Nachfärbung mit Bismarckbraun oder nach Weigert mit Vorfärbung mit Lithionkarmin. Beide Methoden gaben gleich gute Resultate.

Die Schnitte zeigen, dass der Belag aus einem nekrotischen, einige Fibrinfasern und Leukncyten enthaltenden Gewebe besteht. Die Leukocyten liegen

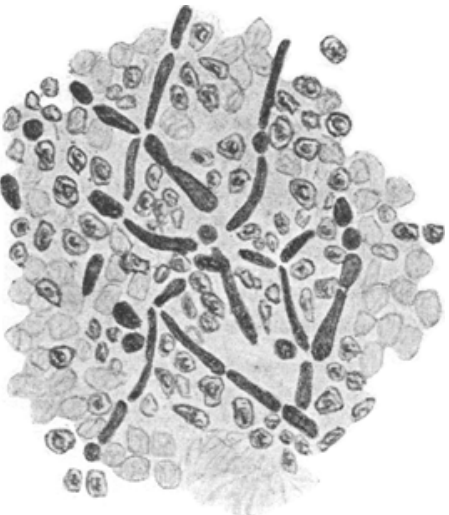

Fig. 3.

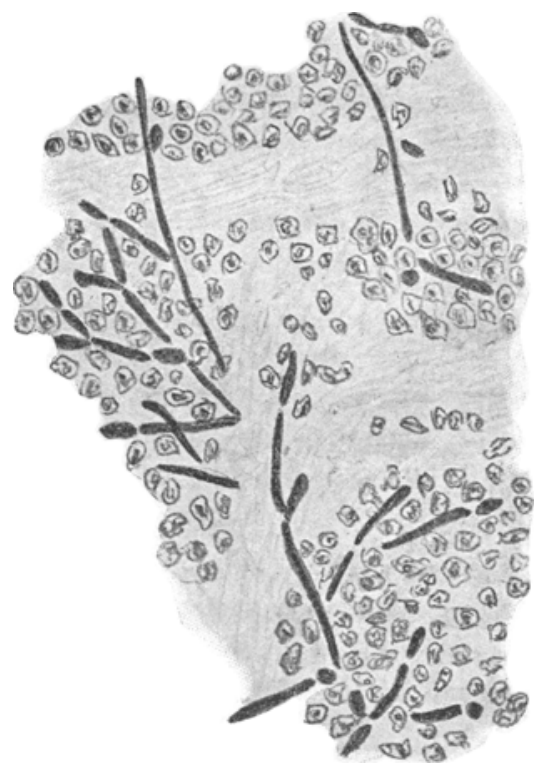

Fig. 4. oft in langen breiten Zttgen zusammen, die dann regelmässig in ibrer Mitte in kleineren oder grösseren Mengen Pilzfäden oder Conidien enthalten. Die Fäden bilden zuweilen ein dichtes, unentwirrbares Mycellager, in dem eine Menge Conidien liegen, zuweilen gehen sie stern- 
förmig von einem Punkte aus und sehen so mit ihren vielfachen Verästelungen den Schneefiguren ähnlich. Weiter finden sich kleine Sprossverbände; kurz die Bilder sind so mannigfaltig, dass sie sich nicht einzeln beschreiben lassen (Fig. 3 und 4). Die Conidien allein finden sich mehr an der Oberfläche des Geschwürs in grösseren Haufen, oder auch vereinzelt in dem nekrotischen Gewebe (Fig. 5). Auf dieses folgt ein zellreiches, junges Bindegewebe, in dem neben Fibroblasten und feinster Intercellularsubstanz auch vielgelappte Leukocyten liegen. Das Gewebe wird dann weitmaschiger, reicher an Fibrillen und ärmer an Zellen, die aber noch immer ihre Sternform beibehalten, und weist ausserdem eine grosse Anzahl von Mastzellen auf.

Es sind nun noch die in dem Sectionsprotokoll erwähnten Veränderungen der Wandung der Flexur näher zu betrachten. Es handelt sich um ein kaum $10 \mathrm{~cm}$ langes Stuck des S. romanum, dessen Wand mit den Appendices epiploicae stellenweise

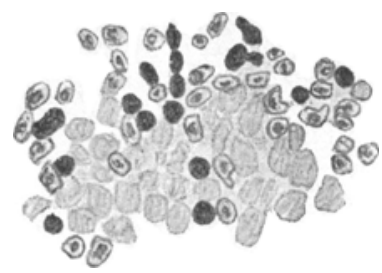

Fig. 5. bis zn $3 \mathrm{~cm}$ verdickt ist. Die Verdickung ist keine ganz gleichmässige, sie betrifft nur einen Theil des Querschnittes und geht ganz allmählich in die normale Wandung tiber, macht aber von aussen betrachtet durchans den Eindruck eines derben Tumors. Auf Schnitten nach den verschiedensten Richtungen hin sieht man, dass die Mucosa und die Submucosa vollkommen unverändert und auf der mässig verdickten Muscularis verschieblich sind. Die Veränderung betrifft also im Wesentlichen die Subserosa und Serosa. Jenseits der Muscularis ist das Grundgewebe ein sehr weitmaschiges, rötbliches bis braunes, das in breiten, sich vielfach verästelnden Zitgen kleine Mengen von Fettgewebe umgiebt. Dazwischen liegen bis hanfkorngrosse Eiterherde, von denen besonders die grösseren einen grünlichen Farbenton haben.

Jene breiten Gewebszilge erweisen sich unter dem Mikroskop zum grössten Theil als beginnende Nekrosen. Es ist ein feines Netzwerk vielfach verschlungener Fasern, das ohne die Reaction desselben zu geben, dem Fibrin ansserordentlich ähnelt. In feinen Maschen sieht man nur einzelne, sich schlecht färbende und in ihrer Gestalt auch schwer zu erkennende Zellen und einzelne Pigmentkörnchen. Die Nekrose scheint immer in der Mitte der Züge zu beginnen, während zu beiden Seiten ein sehr zellreiches, junges Bindegewebe vorherrscht, das kleine Haufen von Fettzellen umgiebt und zwischen die einzelnen Fettzellen eindringt. Neben ausgedehnten Hämorrhagien sieht man in ihm strotzend gefüllte Gefässe und Capillaren, die auch zwischen den Fettzellen sichtbar sind. An einzelnen Stellen kommt es zu stärkerer Anhäufung von Leukocyten, die oft scharf umschriebene Eiterherde mit centralem Zerfall bilden, in denen sich mit grösster Regelmässigkeit jener Fadenpilz in der bereits mehrfach beschriebenen Form vorfindet. An einer Stelle der Darmwand fand ich auch eine kleine Lymphdruse, in der vereinzelte Conidien lagen. Die Zellen der Serosa sind stark gewuchert; sie bilden ein breite Zone, in der sich kleine Eiterherde mit Fäden und Conidien befinden. Unter ihr 
Ein Fall von Soorinfection als Beitrag zur Pathogenese des Soor. 553

liegt eine breite Schicht echten Granulationsgewebes mit vielen neugebildeten Gefässen, das allmählich in jenes oben beschriebene, in Nekrose begriffene Gewebe thbergeht.

Leider wurden weder die beiden verkästen Lymphdrüsen aus der Gegend des Coecum noch auch Theile der infiltrirten linken Lunge nach der Section zur mikroskopischen Untersuchung zurückbehalten.

Es wurde bereits erwähnt, dass in dem den Belägen der Darmwandung entnommenen Eiter in sehr reichlicher Menge befenartige Zellen ohne Andeutung von Fadenbildung, dagegen keine Spaltpilze gefunden wurden. Dementsprechend lieferten auch die auf Agar hergestellten Strichculturen, zu denen das Impfmaterial ebenfalls von der Darm- resp. Divertikelwand entnommen wurde, Reinculturen jener mikroskopisch nachgewiesenen Hefe, als welche die Krankheitserreger anfänglich angesehen wurden. Indess liessen die daraufhin untersuchten Schnitte keinen Zweifel darüber, dass wir es mit einem Fadenpilz zu thun hatten. Um ihn zu identificiren, machte ich von ihm Züchtungen auf den versehiedensten Nährböden und auch einige Impfungen, deren Resultate ich im Folgenden ausführlich wiedergebe.

\section{Verhalten in den Reinculturen.}

Das frisch bereiteten Bouillon-Reinculturen entnommene Material wies sehr verschieden grosse, die Grösse von rothen Blutkörperchen meist tibertreffende, etwas ovale Zellen auf, die von mehr oder weniger breiter, stark lichtbrechender Membran umgeben sind und ein oder mehrere Kernkörperchen ähnliche Körnchen enthalten. Sie liegen meist in Sprossverbänden zusammen und bilden so auch grössere Ketten, oder es liegen mehrere kleinere einer grösseren an. In alten oder aber stark verdünnten Bouillonculturen wachsen diese Zellen zu langen deutlich septirten Fäden aus, die sich baumästig vertheilen und oft in einem unentwirrbaren, mit Conidien untermischten Mycelhaufen zusammenliegen.

Das Wachsthum geht bei Zimmertemperatur, sehr viel schneller aber bei $35^{\circ} \mathrm{C}$. vor sich. In der Bouillon bildet sich schon nach 24 Stunden ein leichter, weisser, flockiger Bodensatz, der später eine eiterähnliche, compactere Beschaffenheit annimmt, so dass er geschüttelt in langen Wolken aufwirbelt und der Flïssigkeit schliesslich ein getrubtes Aussehen verleiht.

Strichculturen auf Agar und Glycerinagar erscheinen sehr uppig, von etwas gelblich-weisser Farbe und rahmiger Consistenz, und bestehen nur aus Conidien.

Anf Blutserum wird der Strich durchsichtig und perlschnurartig, einzelne Colonieen erreichen nur die Grösse eines Stecknadelknopfes. An älteren Röhrchen tritt bisweilen ein rasenartiges, mit kleinen Ausläufern in die Umgebung übergehendes Wachsthum auf. An letzteren Stellen finden sich neben den Conidien auch einzelne Fäden.

In gewöhnlicher Nährgelatine nehmen die Culturen auf Platten ein 
knopfförmiges Aussehen an und erscheinen unter dem Mikroskop maulbeerartig. Eine Verflüssigung tritt selbst bei Monate alten Culturen nicht ein. Bei Stichculturen entwickeln sich zunächst im Stich kleine runde Colonieen, von denen ans am 2. Tage feine wurzelförmige, sich wieder

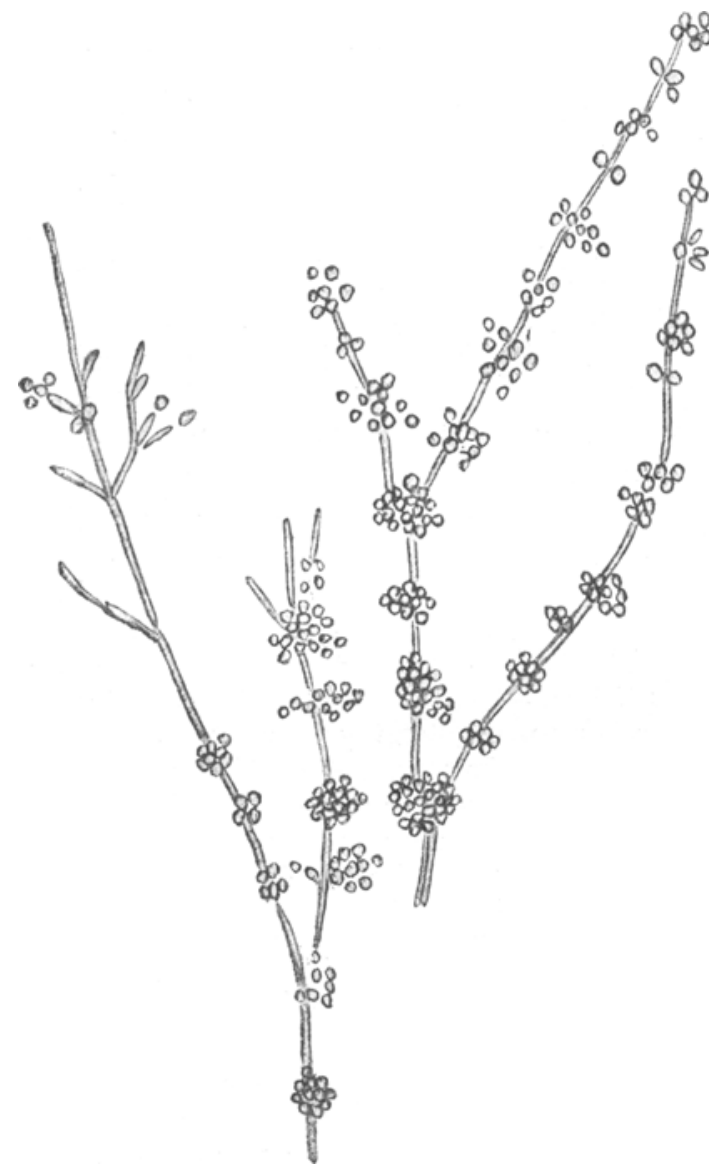

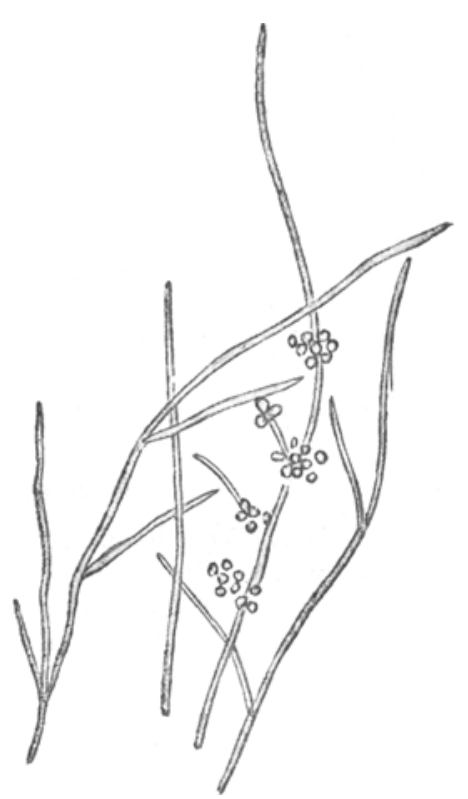

Fig. 7 .

Fig. 6.

theilende Fortsätze in radiärer Richtung und von oben nach unten abnehmend entstehen. Die Oberfläche des Stiches wird von einer knopfförmigen Colonie eingenommen. Das Ganze bietet ein ungemein zierliches Bild, und wenn man den Stich in der von Beneke angegebenen Weise unmittelbar entlang dem Glase anlegt, so ist man im Stande, anch bei stärkerer Vergrösserung, die einzelnen Fasern zu verfolgen. Dieselben bestehen aus etwas von einander stehenden, gegen das Ende hin kleiner werdenden Colonieen, die durch einzelne Fäden mit einander verbunden sind. Das Ende des Ganzen bildet ebenfalls ein Faden mit vereinzelten Seiten- 
sprossen, der deutlich septirt ist. An vereinzelten Stellen des Stiches dagegen und zwar nach der Oberfläche za herrscht die Fadenbildung vor. Man kann dies schon mikroskopisch an der Feinheit der Fasern und deren büschelförmigem Auftreten sehen. Mikroskopisch zeigen die sehr langen Fäden deutliche Septa. Von den Septen aus gehen feinere, sich wiederum theilende Fäden, deren Endglieder kolbig aufgetrieben sind, während das äusserste Ende von einer auffällig grossen, kugelrunden Conidie gebildet wird. Auch zwischen diese Fäden sind kleinste Colonieen eingeschaltet, wie denn tiberhaupt beide Arten des Wachsthums in einander ubergehen. Eine genauere mikroskopische Untersuchung machte ich dadurch möglich, dass ich die Gelatine in Formalin härtete und dann mit dem Gefriermikrotom schnitt. Von den dadurch gewonnenen Bildern füge ich einige Zeichnungen (Fig. 6 und 7) bei.

Ein ganz ähnliches Verhalten in Platten- und Stichcultur findet sich in saurer Gelatine, nur ist das Wachsthum ein schnelleres. Die Stiche unterscheiden sich nur insofern von den vorhergehenden, als die Wurzelfasern nicht gleichmässig in dem ganzen Stich, vielmehr bitschelförmig und in kleinen Abständen auftreten.

In Bierwutrzgelatine ist das Aussehen der Culturen im Stich und auf den Platten wiederum ein ungefähr gleiches, doch treten erst nach Wochen vereinzelte und kurze, seitliche Fortsätze auf. Die Verflussigung beginnt nach $8-10$ Tagen.

Schliesslich zeigte der Pilz die Fähigkeit, Dextrose, Lävulose, Maltose und Lactose zu vergähren, Saccharose dagegen nicht.

\section{Impfresultate.}

Die auf das culturelle Verhalten gegründete Annahme, dass es sich in unserem Falle um Soor handle, suchte ich nun weiter durch Impfversuche zu stïtzen.

Ich injicirte nach dem Vorgange von Grawitz ${ }^{1}$ ) Kaninchen einige Cubikcentimeter einer Bouillonaufschwemmung jenes Pilzes in den Glaskörper und fand nach 6 Tagen das Auge gefüllt mit kleinen gelblichen Flöckchen, die z. Th. der Retina membranartig auflagen und nur an den Einstichöffnungen in festerem Zusammenhange mit ihr standen. Aussen an der Einstichöffnung lag ein knopfförmiger, derber Pilzrasen. Während in der dazu verwandten Aufschwemmung nur Conidien vorhanden waren, zeigten sich im Auge neben grossen Sprossverbänden lange Mycelien mit gruppenweise anlagernden Conidien und Endknospen, wie sie sich in ganz ähnlicher Weise in dem Belag des perforirten Geschwürs fanden.

Weiter impfte ich nach dem Vorgange von ('laut ${ }^{2}$ ) jenen Pilz in die Kropfschleimhaut junger Tauben. Nach 6 Tagen sah man daselbst vereinzelte kleine Beläge, die aus einem Gewirr von Fäden bestanden.

1) Grawitz, Beiträge zur systematischen Botanik der pflanzlichen Parasiten. - Virchow's Archiv. Bd. LXX.

2) Plaut, Neue Beiträge zur systematischen Stellung des Soorpilzes in der Botanik. - Leipzig 1857. 
Besonders reichlich aber zeigten sich dieselben an der Oberfläche der jungen Narbe.

Aus diesen Befunden scheint es mir ausser allem Zweifel, dass wir es nach einem Vergleich der Beschreibungen von Grawitz ${ }^{1}$ ), Plaut ${ }^{2}$ ) und Fischer und Brebeck ${ }^{3}$ ) mit dem Soorpilz und zwar der verflüssigenden Form desselben zu thun haben. Dabei ist mir nur der eine Unterschied im biologischen Verhalten des Pilzes aufgefallen, dass derselbe nämlich im Stande ist, auch Lactose zu vergähren. Nach Fischer und Brebeck, sowie Linossier und Roux ${ }^{4}$ ) kommt dem Soor diese Fähigkeit nicht zu. Doch möchte ich bemerken, dass ich bei Anlegung aller Culturen Controlculturen von echtem Soor herstellte, die durchweg dieselben Resultate auch in Bezug auf die Vergährung der Lactose lieferten; auch bei den Impfversuchen ergaben die Controlversuche mit Soor jedesmal die gleichen Resultate.

Für mich war es nun nach Constatirung dieser Befunde von grösstem Werth, zur weiteren Erklärung unseres Falles festzustellen, wie weit dem Soorpilz und demnachi auch dem hier gefundenen Pilz pathogene, speciell eitererregende Wirkung zukommt.

Im Allgemeinen gilt ja der Soor als ein ziemlich harmloser Pilz, der nicht einmal im Stande ist, kräftige Individuen zu befallen, sondern nur auf der Schleimhaut stark geschwächter und beruntergekommener Kranken sich anzusiedeln vermag. Nur insofern ist sein Erscheinen ein prognostisch ungünstiges Zeichen. Immerhin sind in der Literatur auch einige wenige gegentheilige Fälle bekannt geworden, und besonders haben genauere anatomische Untersuchungen ergeben, dass ihm entschieden lgewisse jpathogene, Wirkungen auf die umgebenden Gewebe zukommen.

So (wiesen Virchow ${ }^{5}$ ) und Wagner ${ }^{6}$ ) darauf hin, dass die Soorfäden in das submucöse Gewebe des Oesophagus eindringen

1) 1. c.; und: Ueber die Parasiten des Soors, des Favus und des Herpes tonsurans. - Virchow's Archiv Bd. CIII.

2) l. c.

3) Fischer u. Brebeck, Zur Morphologie, Biologie und Systematik der Kahmpilze, der Monilia candida Ha n sen u. des Soorerregers. - Jena 1894.

4) Linossier et Roux, Sur la fermentation alcoolique et la transformation de l'alcool en aldihyde provoqués par le champignon du muquet. _Compt. rend. de l'Acad. des sciences de Paris Vol.60. 1890.

5) Virchow's Handbuch der speciellen Pathologie und Therapie.

6) Wagner, Jahrbuch für Kinderheilkunde. Bd. I. 
können, und weiter betonten besonders $\mathrm{Schmidt^{1 } )}$ und Heller ${ }^{2}$ ) das gleiche Verhalten. Sie beschrieben als fast constanten Befund eine bisweilen sehr reiche kleinzellige Infiltration in der Umgebung der Soorfäden und darüber hinaus, Eindringen in Blutgefässe, die dadurch zur Thrombosirung gebracht werden, und eine Neigung des Gewebes zur Nekrose, die Heller z. Th. auf die Circulationsstörung, aber auch auf die directe Wirkung des Soorpilzes zurückführt. Die Neigung zur Thrombose beim Hineinwachsen der Fäden in die Gefässe erklärt den Umstand, dass Metastasen bisher nur in vereinzelten Fällen beobachtet wurden. So fanden Zenker ${ }^{3}$ ) und Ribbert ${ }^{4}$ ) bei mit Soor des Rachens behafteten Individuen multiple Eiterherde im Hirn, in denen sich Soorfäden nachweisen liessen. Ribbert suchte dabei vergeblich nach anderen Organismen. Und schliesslich wies S c h m orl ${ }^{5}$ ) bei einem an Typhus gestorbenen und mit Soor des Oesophagus und Rachens behafteten Kinde bacteriologisch den Pilz in Milz und Niere nach, in welch' letzterer in Mitten kleiner Abscesse neben anderen Mikroorganismen auch der mikroskopische Nachweis von Soor gelang. Erwähnen wir ferner noch, dass Langerhans ") eine durch Soor bedingte, eitrige Entzündung der Oesophagussehleimhaut beschrieb, die er auf chemische Umsatzproducte des Pilzes zurückzuführen geneigt ist, und dass Grasset ${ }^{7}$ ) einen dem Soor in seinem morphologischen Verbalten durchaus gleichenden Pilz in einem Zahnabscess fand, so haben wir im Wesentlichen das für uns Wichtige aus der Literatur über die Pathogenese des Soor beim Menschen wiedergegeben.

Ich möchte nicht verfehlen, darauf hinzuweisen, dass von Tok is $\left.\mathrm{kige}^{8}\right)$ und Gilchrist ${ }^{9}$ ) Oidien als Erreger einer in Knötchenform

1) Schmidt, Ueber die Localisation des Soorpilzes in den Luftwegen und sein Eindringen in das Bindegewebe des 0esophagus. - Beiträge zur patholog. Anatomie und allg. Pathologie 1890. Bd. VIII.

2) Heller, Beitrag zur Lehre vom Soor. Deutsches Archiv für klinische Medicin 1895. Bd. LV.

3) Jahresbericht der Gesellschaft für Natur- u. Heilkunde. Dresden 1860-61.

4) Sitzungsberichte der niederrheinischen Gesellschaft in Bonn 1869.

5) Schmorl, Ein Fall von Soormetastase in der Niere. - Centralblatt für Bacteriologie und Parasitenkunde 1890. Bd. VII.

6) Langerhans, Ein Fall von Soor des Oesophagus mit eitriger Entzündung der Schleimhaut. - Virchow's Archiv Bd. CIX.

7) Grasset, Etude d' un champignon pyogène parasite de l'homme. Archives de médecine expérimentale 1893. I. 3.

8) Tokiskige, Ueber pathogene Blastomyceten. - Centralblatt für Bacteriologie und Parasitenk. 1896. Bd. XIX.

9) Gilchrist, the presence of an oidium in the tissues of a case of pseudolupus vulgaris. - Bulletinof the John Hopkins Hospital. Vol VII Nr.64. 
auftretenden Hauterkrankung, von dem ersteren bei Pferden und Rindern, von dem letzteren beim Menschen beschrieben sind. In diesen Fällen fanden sich in dem Eiterinhalt der Knötchen wie in dem Gewebe nur Conidien, die erst in den Culturen zu Hyphen auswuchsen. Soweit ich aus den Beschreibungen ersehen kann, stimmen die von ihnen gefundenen Oidien auch sonst nicht mit dem unsrigen überein; von dem von Gilchrist beschriebenen geht dies schon daraus hervor, dass dem Pilz jede Fähigkeit der Vergährung von Zuckerlösungen, auch der Dextrose, abgeht.

Während die oben erwähnten Angaben dafür sprechen, dass dem Soor gewisse pathogene Eigenschaften zukommen, steht Klemperer ${ }^{1)}$ - und auch Schmorl vertritt die gleiche Ansicht - auf dem Standpunkt, dass der Soorpilz bei Ansiedelung in inneren Organen niemals eitrige Entzündung resp. Abscesse zu erzeugen im Stande sei. Die zum Nachweis der Wirkungsweise des Soor auf die Gewebe angestellten Versuche ergaben verschiedene Resultate: Klemper er fand bei Injection von Reinculturen in die Blutbahn von Kaninchen eine Mycosis sämmtlicher Organe ohne entzündliche Reaction, Steiner ${ }^{2}$ ) dagegen fand bei gleichen Versuchen um die Soorcolonieen herum kleinzellige Infiltration, die er als directe Wirkung des Soorpilzes bezeichnet, und ebenso sah Ernst ${ }^{3}$ ) in den Nieren eines nach der Injection gestorbenen Kaninchens zahlreiche eitrig aussehende Herde, die mikroskopisch als Nekrose der Harnkanälchen, peritubuläre Infiltration und Wucherung des Pilzes zu erkennen waren.

Ich habe nun auch selbst zur Entscheidung dieser Frage mit dem uns beschäftigenden Pilz einige Versuche an Thieren gemacht und diese Versuche wie zuvor durch in ganz gleicher Weise vorgenommene Impfungen mit Soor, den ich auf andere Weise erlangt hatte, controlirt. Ich benutzte Aufschwemmungen frischer Agarculturen in Bouillon, von denen ich Kaninchen und Meerschweinchen subcutan oder intramusculär einıge Cubikmillimeter einspritzte. In allen Fällen bildete sich an der Impfungsstelle ein bis zum 3. Tage sehr langsam sich vergrössernder Knoten, der in den nächsten Tagen unverändert bestehen blieb und, falls das Thier nicht getödtet wurde, allmählich verschwand. Bei den am 6.-7. Tage getödteten Thieren war jener Knoten regelmässig sehr scharf umschrieben, etwa kirschkern- bis

1) Klemperer, Ueber die Natur des Soorpilzes. - Centralbl. für klinische Medicin 1895 .

2) Steiner, Beiträge zur Pathogenese des Soorpilzes. - Centralblatt für Bact u. Parasitenk. 1897. Bd. XXI.

3) Ernst, Ueber eine Nierenmykose und das gleichzeitige Vorkommen verschiedener Pilzformen bei Diabetes. - Virchow's Archiv. Bd. CXXXVII. 
kirschgross und zeigte in der Mitte vollkommen eitrigen Zerfall des Gewebes, während die Umgebung den Abscess fast kapselartig einschloss und nur wenig entzündliche Erscheinungen aufwies. Aus dem Eiter habe ich in allen Fällen wiederum Reinculturen des Pilzes erhalten. Mikroskopisch fanden sich alle schon oben beschriebenen Formen darin, speciell kleinere 'Sprossverbände, daneben aber auob lange, mehrfach verästelte, häufig gewundene und mit Endknospen versehene Fäden. Sie trugen aber vielfach Zeichen von Degeneration: ungleichmässige Färbbarkeit, Zerfall in kleine, noch färbbare Körnchen. In Schnittpräparaten sah man abgesehen von dem nur aus Leukocyten und Pilzen zusammengesetzten Centrum an den Randpartien deutliche Abkapselungsprocesse in Form von circulären jungen Bindegewebszügen, die nach dem Abscess zu noch leichte Infiltration aufwiesen. Alle diese Verhältnisse deuten darauf hin, dass der Soorpilz subcutan injicirt wobl geeignet ist, bei Kaninchen unter anfänglicher Fortentwicklung und Auswachsen zu Fäden locale Eiterung zu machen. Dieselbe zeigte aber durchaus keinen progredienten Charakter, vielmehr weisen die degenerativen Veränderungen des Pilzes, die Abkapselung und das allmähliche Verschwinden der Abscesse darauf hin, dass der Pilz für jene Thiere wohl eitererregende, dagegen keine deletären Wirkungen besitzt.

Einige Versuche, die ich ohne Controlversuche nur mit dem von dem Kinde gewonnenen Material gemacht habe, füge ich hier anhangsweise noch mit an.

Ieh injicirte einem Kaninchen durch die ganze Dicke der Bauchmuskeln hindurch eine grössere Menge der betreffenden Aufsehwemmung in den Peritonealraum. Es bildete sich im Verlauf des Stiches eine derbe Infiltration, und als das Thier am 6. Tage, während welcher Zeit es übrigens vollkommen gesund sehien, getödtet wurde, fanden sich ausser einer ziemlich ausgedehnten Bauchdeckeneiterung dem Einstich gegenüber an der Darmwand mehrere bis hirsekorngrosse Knötchen. In der Umgebung derselben war die Wand deutlich verdickt, jedoch ohne sichtbare Zeichen stärkerer Entzündung. Mehrere weitere Herde, die offenbar auf dem Wege der Lymphbahnen entstanden waren, da sie vom Einstich weit ablagen, lagen in oder an der Oberfläche der Leber. Aus allen Abscessen wurden Reinculturen des Pilzes gezogen, und auch mikroskopisch gelang ihr Nachweis überall.

Ein anderes Kaninchen, dem nach dem Bauchschnitt Culturen in die wundgemachten Darmwände in grosser Menge und 'Ausdehnung fest eingerieben wurden, überstand den Eingriff sehr gut, hatte zwar anfänglich einige unbestimmte Resistenzen im Abdomen, war aber noch viele Monate darauf vollkommen gesund.

Deutsch $\theta$ Zeitschrift f. Chirurgie. LXII. Bd. 
Schliesslich wurde einem Kaninchen intravenös $1 \mathrm{qcm}$ Aufschwemmung injicirt. Bei diesem fand sich abgesehen von einem Abscess an der Impfstelle eine ausgesprochene metastatisch eitrige Nephritis. Besonders die Rinde war durchsetzt von zahllosen, stecknadelkopfgrossen, weissen Herden, während im Mark neben einigen runden Herden weissliche Streifen in geringer Menge lagen. An anderen Organen war nichts Auffallendes zu erkennen.

Mikroskopisch bestehen jene Herde aus Leukocyten mit nur ganz vereinzelten epithelioiden Elementen. Sie scheinen ihren Ursprung zum

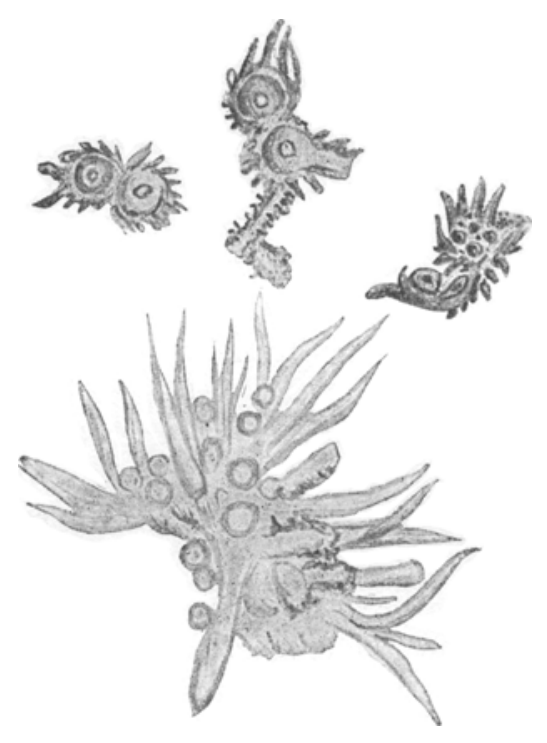

Fig. 8. grössten Theil von dem Glomerulis zu nehmen, denn man bemerkt bisweilen innerhalb der kleinen Abscesse noch Reste von Glomerulusschlingen, während an anderen Stellen in nächster Nähe der Glomeruli die ersten Anfänge kleinzelliger Infiltration zu sehen sind. Dabei zeigen die Reste der Nierensubstanz starke parenchymatöse Veränderungen. Am bemerkenswerthesten ist es, dass sich in Mitten der kleinen $\mathrm{Ab}$ scesse fast überall Mycelien oder Conidien unseres Pilzes finden. Sie sind an einigen Stellen in grossen Haufen mit einander vermischt, so dass es, zumal die Färbung eine durchaus gleichmässige und intensive ist, den Eindruck macht, als hätten sich hier die zur Ausscheidung gekommenen Conidien angesiedelt und vermehrt. An anderen Stellen hingegen zeigen die Fäden, obwohl ebenfalls in Mitten von Abscessen gelegen, degenerative Veränderungen, die sich abgesehen von der mangelhaften Färbbarkeit und dem Zerfall darin zeigen, dass eigenthümliche kolbenförmige und zackige Fortsätze um den Pilz herum entstehen (Fig. 8).

So werden einzelne Conidien von derartigen Gebilden umgeben, oder es entwickeln sich um ein Mycellager Gebilde, die den Aktinomycesdrusen oft sehr ähnlich sind und mit diesen ausserdem das gemeinsam haben, dass sie sich in Pikrinsäure intensiv gelb färben. Diese Fähigkeit geht dem lebensfähigen Pilz ganz ab, nur die „Hüllen der Conidien nehmen bisweilen eine ganz schwach gelbliche Färbung 
an. Lichtheim ') giebt von den pathogenen Mukorineen ganz ähnliche Abbildungen, und auch Sch morl (l. c.) hat bei Soor Bilder gefunden, die der Beschreibung nach mit den meinigen übereinstimmen. Eine sichere Erklärung für diese Bildungen steht noch aus, wahrscheinlich handelt es sich aber doch wohl um Degenerationsvorgänge.

Fassen wir noch einmal kurz unsere Befunde und die Resultate der verschiedenen Untersuchungen zusammen, so hatten wir einen Fadenpilz als Ursache einer Erkrankung gefunden, die, obne in ihrem klinischen Verlauf irgend welche Eigenthümlichkeiten zu zeigen, zum Tode des Kranken geführt hatte. Da ein analoger Fall meines Wissens überhaupt noch nicht in der Literatur mitgetheilt worden ist, so musste mir vor Allem daran liegen, den Fadenpilz zu identificiren. Die auf Grund des gleich nach der Section angefertigten Deckglastrockenpräparates gestützte Annahme, dass es sich um eine Hefe handle, wurde durch die mikroskopische Untersuchung der Schnittpräparate unhaltbar. Dagegen ergaben die Züchtungen und Impfungen Resultate, die, zumal sie durch gleiche Versuche mit zwei Soorculturen controlirt wurden, zu der Annahme drängten, dass der gefundene Pilz mit dem Soor identisch sei. Obwohl mit diesem Beweise, dass es sich um Soor handle, auch der Beweis erbracht war, dass der Soor als pathogener Pilz anzusehen ist, hielt ich es bei den widersprechenden Ansichten der Autoren über die Pathogenese des Soor doch für angezeigt, seine pathogene Wirkung durch Thierversuche zu prïfen, und gelangte dadurch, obwohl zuzugeben ist, dass weder Kaninchen noch Meerschweinchen in Folge der Impfungen zu Grunde gingen, zu positiven Resultaten, besonders konnte ich zeigen, dass nach intravenöser Injection nicht nur, wie Klemperer angiebt, eine Mykose eintritt, sondern dass es in der Umgebung der mykotischen Herde gleichzeitig zur Eiterung kommt.

Sehen wir nun von den bereits angeführten Literaturangaben über die Pathogenese des Soor im Speciellen beim Menschen ab, so werden meines Wissens nur von Tokiskige und Gilchrist Oidien als Krankheitserreger erwähnt. Ich führte bereits aus, dass dieselben mit unserem Pilz nicht identisch sein können, und möchte nun noch kurz auf den Fall von Grasset eingehen. Dieser wies, wie erwähnt, in einem Zahnabscess einen eitererregenden, dem Soor durchaus gleichenden Pilz nach und sieht in ihm nur deshalb eine neue Pilzart, um auf diese Weise der bisher geltenden Anschaunng über die Gutartigkeit des Soor gerecht zu werden. Er fusst bei dieser Annahme allein darauf, dass es Linossier und Roux bei intra-

1) Lichtheim, Ueber pathogene Mukorineen. - Zeitschr. f. klin. Med. 1884. 
venöser Injection des Soor nur gelungen sei, eine Mykose der parenchymatösen Organe ohne Eiterung zu erzeugen, während er mit seinem Pilz wohl subcutane Eiterung, aber keine Mykose erzeugte. Zum Schluss giebt er allerdings zu, dass man zwei Hypothesen aufstellen könne: entweder sei der im Abscess gefundene Pilz eine besondere Art, was noch zu entscheiden sei, oder es sei Soor, der in Folge anormaler Lebensbedingungen aussergewöhnliche Eigenschaften erworben habe. Die von mir angestellten Untersuchungen ergaben indess, dass der Soor sehr wohl subcutane Eiterung machen kann, und ebenso sind die Befunde von Zenker und Ribbert, die in metastasischen Abscessen beim Menschen Reinculturen von Soor fanden, so einwandfrei, dass wir dem Soor eitererregende Wirkung zuschreiben müssen. Damit ist aber jeder Grund, in dem Grasset'schen Fall eine besondere Art anzunehmen, hinfällig. Die Bedeutung des Falles für die Pathogenese des Soor verliert allerdings dadurch vollkommen an Werth, dass Grasset ausser jenem Fadenpilz auch noch andere Mikroorganismen fand, denen die Entstehung der Eiterung zugeschrieben werden kann.

Es liegt wohl nahe, an dieser Stelle auch auf die Befunde einzugehen, die Busse') und Buschke ${ }^{2}$ ) bei der Hefemykose des Menschen gemacht haben, und sie mit den pathologisch-anatomischen Bildern unseres Falles zu vergleichen. Makroskopisch bieten beide gewisse Aehnlichkeiten: hier wie dort finden wir tumorartige Bildungen, die auf dem Durchschnitt weiche, an Käse erinnernde Massen enthalten. Während diese Massen indess bei der Hefeinfection eine Beschaffenheit haben, die sich schwer unter eine der bekannten Nekrosen einreihen lässt, so konnten wir den Inhalt der Herde in unserem Fall schon makroskopisch als dick consistenten Eiter bezeichnen, der allerdings eine etwas grünliche Färbung hatte. Ziehen wir auch die Thierversuche von Busse zum Vergleich heran, so muss es auffallen, dass er bei der Impfung von Hefe in die Bauchhöhle von Mäusen tumorartige, riesenhafte Verdickung allen Fettgewebes in der Bauchhöhle, speciell der Appendices epiploicae fand, wie wir es auch in unserem Fall constatiren konnten. Während indess in den Fällen von Busse - wir kommen damit auf das mikroskopische Verhalten beider Arten von Infection - nur geringe Zeichen von entzündlicher Infiltration vorhanden waren, die Verdickung vielmehr allein auf eine massenhafte Invasion von Hefezellen zu beziehen

1) Busse, Die Hefen als Krankheitserreger. - Berlin 1897.

2) Buschke, Ueber Hefemykosen bei Menschen und Thieren. - Sammlung klinischer Vorträge 1898. 
war - ein Verhalten, das auch in der Beobachtung am Menschen mehrfach von beiden Autoren betont wird - so treten bei uns die entzündlichen Erscheinungen bis zur eitrigen Einschmelzung des Gewebes durchaus in den Vordergrund. Ueberall, wo wir den Soor in kleineren Häufchen fanden, bildete er das Centrum eines Herdes von Eiterkörperchen, an Stellen aber, wo die Eiterung schon weiter vorgeschritten ist, zeigte sich ganz wie bei Infectionen mit den bekannten Eitererregern centrale Nekrose mit einem grösseren entzündlichen Hofe. Diese reactive Entzündung, die in unserem Fall die alleinige Ursache der tumorartigen Bildung ist, greift indess in weit ausgedehnterem Maasse auf das umgebende Gewebe über, als es bei sonstigen Eiterungen der Fall ist, und wie wir es in ähnlicher Weise wohl nur in dem Peritonealraum, speciell am Netz als Folge einer Infection kennen. Da wir aber in letzterem Fall eine Analogie mit anderen Infectionen haben, so bleibt ïberhaupt die Frage offen, ob die tumorartige Verdickung am S. romanum, die ja im Wesentlich en nur Serosa und Subserosa betraf, eine specifische Wirkung des Fadenpilzes sei oder nur eine specifische Reaction des Peritoneum auf Reize, die es treffen, überhaupt.

Aus alledem geht hervor, dass die Hefemykose und die Soorinfection von einander wesentlich verschiedene pathologisch-anatomische Veränderungen zeigen, und ich kann es deshalb im Gegensatz zu Buschke nicht für angezeigt halten, Blastomyceten und Oidien gemeinsam mit einander abzuhandeln.

Ich bin auf diese Vergleiche der durch Hefen und Oidien hervorgerufenen Krankheitsbilder nur deshalb näher eingegangen, um zu zeigen, dass die Verwandtschaft beider Pilze im System allein nicht zu der Annahme genügt, dass auch die pathologisch-anatomischen Veränderungen, die sie bedingen, die gleichen seien. Da wir es aber bei den Oidien mit einer ausgesprochenen Entzündung zu thun haben, so bedarf es auch keines weiteren Eingehens auf die reichhaltige Literatur über Blastomykosen und ihre Beziehung zu echten Tumoren.

Gehen wir nun auf die Entstehungsursache des ganzen Krankheitsprocesses in unserem Falle näher ein, so ist das Vorkommen des Soor im Darm an 'und für sich eine Seltenheit. Zwar lässt sich die ältere Anschauung, dass die mit Cylinderepithel bedeckten Schleimhäute gegen das Haften von Soor immun mache, nicht mehr halten, denn es sind in der Beziehung mehrere gegentheilige Befunde gemacht worden. So berichtet Zaleskyl) über einen Fall von Soor

1) Zalesky, Ein Fall von Soor im Magen. - Virchow's Archiv Bd. XXXI. 
im Magen, in dessen Epithelialschicht sich einzelne Pilzfäden und Sporen fanden, Parrot ${ }^{1}$ ) sah Soorwucherungen im Cöcum, und Heller (l. c.) solche auf dem Grunde eines alten Magengeschwürs. Derselbe fand in einem anderen Fall das Epithel der Luftröhre glatt von den Soorfäden durchwachsen. Immerhin bleiben diese Befunde anscheinend vereinzelt, und das ist um so auffälliger, als bei reichlichen Soorwucherungen im Rachen und Oesophagus isich oft lebensfähige Conidien im Stuhlgang nachweisen liessen. Da Anhaltspunkte dafür, dass das Kind früher Soorwucherungen an anderen Stellen des Körpers, speciell im Rachen gehabt hat, fehlen, so müssen wir wohl annehmen, dass der Halstheil des Divertikels der primäre Sitz der Erkrankung war, in den der Pilz vom Darmlumen aus hineingelangte. Ob hier bereits schon vorher ein ulceröser Process bestand, der das Haften des Pilzes begünstigte, lasse ich dahin gestellt. Anhaltspunkte dafür haben wir in dem pathologisch-anatomischen Bilde des Geschwürs nicht. Es ist mit gleicher Wahrscheinlichkeit anzunehmen, dass die schwielige Umwandlung der Umgebung des Geschwürs und damit auch die Stenose im Halstheil des Divertikels eine Folge des durch den Soor hervorgerufenen ulcerösen Processes war. Dieser sowobl wie der Befund der verkästen Drüsen in der Gegend des Cöcum sprechen dafür, dass es sich um einen recht chronisch verlaufenden Process handelte, denn wir werden kaum fehlgehen, wenn wir die verkästen Drüsen, obwohl sie nicht untersucht wurden, als Metastasen ansehen, da sie der Lage nach etwa dem Lymphnetz des Divertikels entsprachen, und wir ferner aus dem Befunde mehrerer Conidien in einer Lymphdriise der Darmwand zn dem Schluss berechtigt sind, dass die Verbreitung des Pilzes auf dem Wege der Lymphbahnen thatsächlich stattfand. Weiter ist anzunehmen, dass sich im Anschluss an die Stenose im Halstheil der Hydrops des Divertikels entwickelt hat, der seinerseits, wie die Anhäufung von Pigment in dem schleimigen Inhalt lehrt, zu Entzündungen und damit zu Verklebungen mit den umliegenden Darmschlingen führte. Die Perforation des Geschwürs trat am 4. Tage vor der Aufnahme, an dem sich die ersten Symptome einer umschriebenen Bauchfellentzündung entwickelten, ein, und es kam nun zu einer Dissemination des Pilzes in einen durch die früheren Verklebungen abgeschlossenen Raum, aber auch zu einer Weiterverbreitung in den Wandungen der anliegenden Darmschlingen. Es ist bekannt, wie schnell es in den von Serosa bedeckten Theilen des Abdomen in Folge einer Infection durch fibrinöse Ausscheidungen, Desquama-

1) Parrot, Clinique des nouveaux nés. 
tion des Endothels und Auswanderung von Leukocyten zu selbst tumorartigen Auftreibungen kommen kann, und da das Aussehen der Gewebe in der Wandung der Flexur und der Blase dieser Auffassung durchaus nicht widerspricht, die Zellen vielmehr überall ein junges Aussehen haben, so werden wir auch rom anatomischen Standpunkte aus an der Auffassung festhalten können, dass die Perforation an jenem Tage erfolgte.

Es würde wunderbar sein, wenn wir nicht auf den Wandungen der Darmschlingen, die den Abscess begrenzten, auch Spaltpilze gefunden hätten. Wir fanden sie in kleinen Haufen und einzeln, allerdings nur in ganz verschwindender Menge, der Oberfläche anliegend, und können uns das Fehlen derselben in den gleich angelegten Culturen nur dadurch erklären, dass sie von den Soorculturen sofort überwuchert wurden. In Folge ihres Nachweises könnte höchstens die Frage aufgeworfen werden, ob die Ursache des Todes, der als Folge der Herzschwäche durch Infection anzusehen ist, nicht sowohl auf die Einwirkung des disseminirten Soorpilzes als vielmehr auf die Umsatzproducte dieser Spaltpilze zurïckzuführen ist. Eine grössere praktische Bedeutung kommt dieser Frage nicht zu. Die Thatsache, dass in den Abscessen der Wandung des S. romanum nur der Fadenpilz nachzuweisen war, und dass also die pathologischen Veränderungen innerhalb derselben nur auf ihn zurückzuführen sind, bleibt bestehen, und hierin liegt der Hauptwerth des uns beschäftigenden Falles. 